\title{
Capillary leak syndrome and disseminated intravascular coagulation after kidney transplantation in a patient with hereditary angioedema - A case report -
}

\author{
Jeong Wook Park ${ }^{1,2}$, Jinyoung Seo', Sang Hun Kim ${ }^{1,3}$, and \\ Ki Tae Jung ${ }^{1,3}$
}

'Department of Anesthesiology and Pain Medicine, Chosun University Hospital, ${ }^{2}$ Department of Medicine, Graduate School, Chosun University, ${ }^{3}$ Department of Anesthesiology and Pain Medicine, School of Medicine, Chosun University, Gwangju, Korea

Received December 22, 2020

Revised January 10, 2021

Accepted January 12, 2021

\section{Corresponding author}

Ki Tae Jung, M.D.

Department of Anesthesiology and Pain Medicine, Chosun University

Hospital, 365 Pilmun-daero, Dong-gu, Gwangju 61453, Korea

Tel: 82-62-220 3223

Fax: 82-62-223-2333

E-mail: mdmole@chosun.ac.kr
Background: Hereditary angioedema (HAE) is a rare disease caused by the deficiency of $\mathrm{C} 1$ esterase inhibitor. HAE has a risk of life-threatening complications such as capillary leak syndrome (CLS) and disseminated intravascular coagulation (DIC).

Case: A 42-year-old male patient with HAE presented for deceased-donor kidney transplantation. Prophylactic fresh frozen plasma (FFP) was given before surgery because of the risk of edema development. With careful management during anesthesia, there were no problems during surgery. However, generalized edema, hypotension, hypoalbuminemia, massive drainage of serosanguineous fluids from the intraabdominal space, and DIC occurred on the day after surgery. CLS was suspected and sustained hypotension with generalized edema became worse despite treatment with albumin, danazol, FFP, and vasoactive drugs. The patient's condition worsened despite intensive care and he died due to shock.

Conclusions: The anesthesiologist should prepare for the critical complications of HAE and prepare the appropriate treatment options.

Keywords: Capillary leak syndrome; Complement C1 inhibitor protein; Complement C1s; Disseminated intravascular coagulation; Hereditary angioedema; Kidney transplantation.
Hereditary angioedema (HAE) is a rare, life-threatening autosomal dominant disorder caused by a deficiency of $\mathrm{Cl}$ esterase inhibitor (C1-INH), with an estimated prevalence of 1:50,000 [1,2]. HAE can be classified by the levels of $\mathrm{Cl}$ INH. Type I is diagnosed by low levels of C1-INH and C, and type II is diagnosed by normal levels but dysfunctional C1-INH $[1,2]$. HAE is potentially fatal because it may present with sudden life-threatening edema of the skin and aerodigestive tract (face, extremities, larynx, genitals, and trunk) recurrently or spontaneously [2,3]. Thus, patients are recommended to avoid general anesthesia with endotracheal intubation, or careful prophylactic therapies before surgery are required if surgery with general anesthesia is unavoidable. Besides the well-known clinical presentations, HAE may also produce hypovolemic shock due to the tissue leakage of fluids [4] and may lead to potentially

This is an Open Access article distributed under the terms of the Creative Commons Attribution Non-Commercial License (http://creativecommons.org/licenses/by-nc/4.0) which permits unrestricted non-commercial use, distribution, and reproduction in any medium, provided the original work is properly cited.

Copyright (C) the Korean Society of Anesthesiologists, 2021 
life-threatening conditions such as capillary leak syndrome (CLS) and disseminated intravascular coagulation (DIC) $[5,6]$. Here, we report a case of suspected CLS and DIC after kidney transplantation in a patient with HAE. The Institutional Review Board approved the study (CHOSUN 202005-005) to publish in a case report and granted a waiver of consent from the patient.

\section{CASE REPORT}

A 42-year-old male patient (height: $165 \mathrm{~cm}$, weight: 61.7 $\mathrm{kg}$ ) was admitted due to acute kidney injury with dyspnea. He complained of swelling in the face and upper arms, which occurred once or twice per year for 30 years. He could not remember his familial history. During admission, the patient's facial swelling got worse and HAE was suspected. According to the laboratory results, the level of $\mathrm{C} 4$ and C1-INH were $7.15 \mathrm{mg} / \mathrm{dl}$ (normal range: 10-40 mg/ $\mathrm{dl}$ ) and $5.0 \mathrm{mg} / \mathrm{dl}$ (normal range: $21-39 \mathrm{mg} / \mathrm{dl}$ ), respectively. The patient was treated with high doses of androgen therapy (Danazol, Young Poong Pharma, Korea; 600 mg daily) as prophylactic maintenance therapy for the swelling due to HAE. However, his kidney injury worsened and progressed to chronic kidney disease. A decision for kidney transplant surgery was made, and he received hemodialysis until surgery.

Seven months later, the patient was selected as the recipient of a kidney from a deceased donor and emergency surgery was planned. However, his level of C1-INH was still low $(15.7 \mathrm{mg} / \mathrm{dl})$, and there was a risk of edema developing during surgery. Thus, three units of fresh frozen plasma (FFP) were administered about one hour before surgery because C1-INH concentrate was not available due to the suspension of imports in Korea, although it was designated as an orphan drug. Anesthesia was induced with thiopental sodium ( $5 \mathrm{mg} / \mathrm{kg}$ ) and cisatracurium $(0.17 \mathrm{mg} / \mathrm{kg})$. Careful intubation by video-laryngoscopy was performed with a $7.5 \mathrm{~mm}$ microcuffed endotracheal tube (Taper Guard $^{\circledR}$, Mallinckrodt, Ireland). The cuff pressure was adjusted to $20 \mathrm{cmH}_{2} \mathrm{O}$ using a cuff manometer (Mallinckrodt) and confirmed air leakage at an airway pressure of more than $20 \mathrm{cmH}_{2} \mathrm{O}$. Anesthesia was maintained with desflurane and remifentanil, and mechanical ventilation was done with a fresh gas flow of $3 \mathrm{~L} / \mathrm{min}$ of an oxygen/air mixture. His vital signs were maintained within $30 \%$ of the baseline during surgery. The surgery took about four hours. The administered fluid and estimated blood loss were 1,000 $\mathrm{ml}$ and $400 \mathrm{ml}$, respectively. At the end of the surgery, neuromuscular blockade was confirmed (train-offour $>99 \%$ ) after the administration of reversal agents (10 mg of pyridostigmine and $0.4 \mathrm{mg}$ of glycopyrrolate). After confirming the absence of edema in the larynx by video-laryngoscopy, early extubation with mask ventilation before restoration of consciousness of the patient was done to avoid irritation of the larynx. The patient recovered consciousness and adequate respiration and was transferred to the aseptic intensive care unit (ICU). Immunosuppressants (tacrolimus and thymoglobulin), ganciclovir, and prostaglandin El were administered according to the hospital protocol. After surgery, his vital signs remained stable and urine output was about $190 \mathrm{ml}$ after reperfusion during the hour in the ICU. During the night, his hemoglobin level remained at $9.4 \mathrm{~g} / \mathrm{dl}$ and there were no abnormalities in the laboratory coagulation tests (platelet count, 202,000; prothrombin time [PT], $11.5 \mathrm{~s}$; activated partial thromboplastin time [aPTT], $20.7 \mathrm{~s}$; and international normalized ratio [INR], 1.04).

The next morning, the patient complained of dyspnea with abdominal discomfort. Then, hypotension (60/30 $\mathrm{mmHg}$ ) and tachycardia (130 beats/min) developed, and he lost consciousness (Fig. 1). He was intubated and mechanical ventilation was applied, and norepinephrine and dobutamine were administered for the treatment of hypotension. At that time, abdominal distension with massive serosanguineous fluids (> 1,000 ml) in the drainage was found and generalized edema with low urine output $(10 \mathrm{ml}$ over eight hours) was also observed. He showed a low hemoglobin level (7.3 g/dl) with coagulopathy (PT, $22.8 \mathrm{~s}$; aPTT, $44.1 \mathrm{~s}$ : and INR, 2.04), and DIC was diagnosed (fibrinogen < $100 \mathrm{mg} / \mathrm{dl}$; fibrin degradation product, FDP $221 \mu \mathrm{g} / \mathrm{ml}$ : and D-dimer, 27,699 ng/ml). Thus, transfusion was performed (red blood cells six units; FFP, three units; cryoprecipitate 10 units: and apheresis platelets, one unit), and continuous renal replacement therapy (CRRT) was applied. Despite the treatments, there was no improvement in his conditions, and an emergency second-look operation was performed with the suspicion of leakage at the anastomosis site. However, there was no anastomosis leakage and normal blood flow to the kidney was confirmed by Doppler. Thus, the operation ended without specific treatment.

However, severe generalized edema with hypoalbuminemia $(2.5 \mathrm{~g} / \mathrm{dl})$ was sustained after surgery, although the $\mathrm{C} 1-\mathrm{INH}$ level had increased to $22.9 \mathrm{mg} / \mathrm{dl}$ after the contin- 


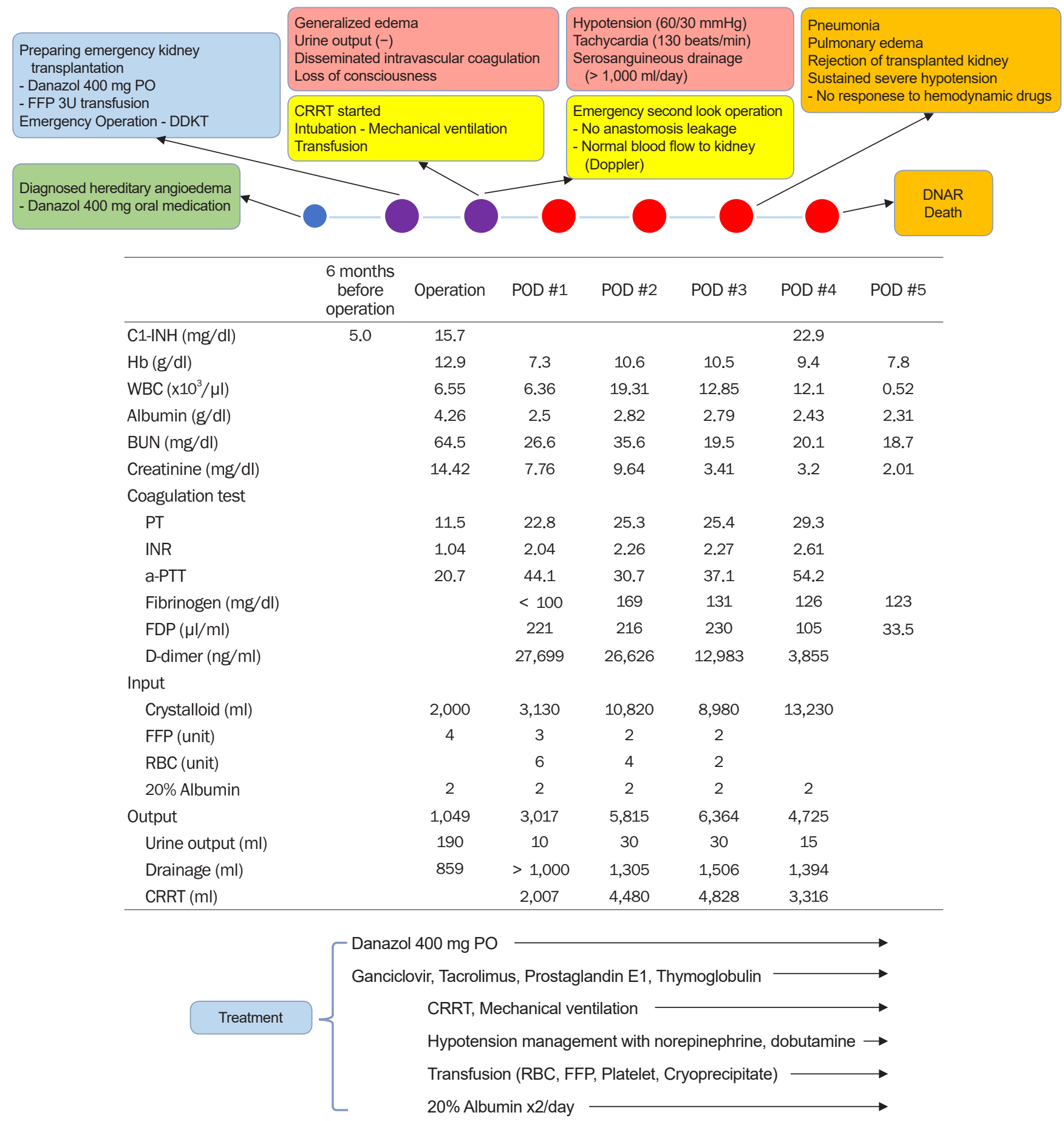

Fig. 1. Course and treatment of the patient after surgery. A patient with hereditary angioedema who was treated with danazol presented for deceased-donor kidney transplantation. Generalized edema with hypoalbuminemia developed the day after surgery and severe hypotension occurred. Despite intensive care, the patient died due to shock. DDKT: deceased-donor kidney transplantation, FFP: fresh frozen plasma, CRRT: continuous renal replacement therapy, DNAR: Do-Not-Attempt-Resuscitation, C1-INH: C1 esterase inhibitor, Hb: hemoglobin, WBC: white blood cells: BUN: blood urine nitrogen, PT: prothrombin time, INR: international normalized ratio, aPTT: activated partial thromboplastin time, FDP: fibrinogen degradation products, RBC: red blood cell, POD: postoperative day. 
uous administration of $20 \%$ albumin, danazol at $400 \mathrm{mg}$ via a Levin tube, and FFP transfusions. We thought that the worsening of the patient's condition despite increases in the C1-INH level was due to harmful substrate proteins in the FFP, and would be transient. The laboratory blood culture results showed no evidence of bacterial growth. Despite intensive care with hypotensive drugs, transfusions, and CRRT, his condition worsened because of sustained generalized edema with hypoalbuminemia, coagulopathy, and DIC. On the fourth day after the surgery, pulmonary edema, pneumonia, and rejection of the transplanted kidney developed and sustained severe hypotension without response to hemodynamic drugs was seen. The patient's guardians did not want any additional treatment and the patient died because of shock after a day.

\section{DISCUSSION}

The complement system contributes to the immunological defense mechanism of the body. HAE is caused by a SERPING1 gene (Serine Protease Inhibitor Gene 1 present on the Long Arm of Chromosome 11 [11q]) defect, which results in decreased or dysfunctional C1-INH [1-3]. Although HAE attacks are unpredictable and the triggering factors are unclear, numerous physical stimuli such as dental and surgical procedures, emotional stress, infections, and medications can be triggering factors of life-threatening angioedema [1-3]. Still, there are no definite perioperative guidelines for the prophylaxis of an HAE attack. However, the following prophylactic therapy is usually suggested: C1-INH concentrate, 1,000 units before surgery; androgens such as danazol (400 to $600 \mathrm{mg} /$ day), five days before and five days after surgery or a procedure; and FFP (two units for adults, $10 \mathrm{ml} / \mathrm{kg}$ in children), one to two hours before surgery [1-3]. Among those treatments, C1-INH concentrate or androgens is known to have the best effect. Tranexamic acid can also be used for long-term prophylaxis, especially in children, but it has not been recommended recently because it has little benefit as short-term prophylaxis or for the acute treatment of HAE attacks due to its delayed onset of action [7]. However, some patients undergo HAE attacks after surgical procedures despite prophylaxis. Thus, careful approaches to the patient with rescue management are required [3].

The patient in our case was diagnosed with type I HAE with low levels of C1-INH and C4, and we used the prophylactic administration of FFP instead of C1-INH concentrate before surgery because it was not available in Korea. Despite prophylactic treatment with FFP, generalized edema developed in the patient, and the catastrophic outcome of DIC developed. We will discuss the possible causes of the deterioration of the patient's condition.

C1-INH plays an important role in the regulation of not only the classical pathway of the immune system but also the plasminogen-plasmin and kallikrein-kinin systems (contact system), and coagulation/fibrinolysis [8]. The deficiency or dysfunction of C1-INH in patients with HAE predominantly results in the increased activation of bradykinin by kallikrein, which leads to vasodilation, an increase in vascular permeability, and the typical angioedema of HAE. In patients with $\mathrm{HAE}, \mathrm{C} 3$ and $\mathrm{C} 1 / \mathrm{C} 1$-inhibitor complexes are easily activated by physical stress [9]. Especially, complement activation is associated with ischemia/reperfusion injury during cold storage of the organ and rejection, and is considered the major factor in graft failure after transplantation by triggering tissue damage and interfering with the anticoagulant and fibrinolytic capacity of the vascular endothelium [10]. Our patient was a kidney transplant recipient with the possibility of increased activation of the immune system. Therefore, it is thought that the regulation of complement activation would not be achieved after major surgery because of C1-INH deficiency in our patient, resulting in the acute rejection of the transplanted kidney by excessive complement activation.

CLS is one of the common complications associated with low C1-INH activity [10]. CLS is characterized by hypotension or multi-organ failure by massive third-space loss of fluids due to increased capillary permeability, which is accompanied by extravasation and diffuse edema. We considered CLS as the cause of the sustained hypotension because of the distinguishing features such as generalized edema with hypoalbuminemia despite albumin treatment, massive drainage of serosanguineous fluids from the intraabdominal space without anastomosis leakage, and the absence of anaphylaxis or sepsis, which should be ruled out due to similar characteristics [11]. Treatment for the prevention of antibody-mediated rejection after kidney transplantation has the potential risk for developing CLS [3].

C1-INH also plays a significant role in both coagulation and fibrinolysis [8]. The activation of C1-INH leads to thrombin generation and plasmin inhibition, which accounts for about $15 \%$ of the fibrinolysis. Hence C1-INH deficiency can lead to pathologic thrombosis. Generally, HAE 
is not associated with coagulation properties clinically. However, there is a possibility that $\mathrm{C} 1$-INH deficiency might affect the coagulation system in the pathologic condition. Kodama et al. [12] revealed that the symptom of an HAE attack in older adults was hypercoagulation because activation of the complement system mainly activates the tissue factor pathway instead of the kallikrein-kinin system. Such physiologic changes with aging lead to the generation of thrombin and the activation of antithrombin-III during an HAE attack, which precipitates DIC. The hypercoagulability of pregnancy and intravascular fluid depletion due to increased vascular permeability during an HAE attack may also contribute to the development of DIC [6]. In our patient, DIC developed simultaneously from the severe intravascular volume depletion in the HAE attack. The decreased hemoglobin level due to DIC was misinterpreted as anastomosis leakage and interfered with the diagnosis of CLS.

In the treatment of acute HAE attacks, C1-INH concentrate, ecallantide (a kallikrein inhibitor) and icatibant (a bradykinin B2-receptor antagonist) are recommended as the first-line drugs [7]. However, as mentioned above, none of those drugs were available in Korea at that time, and it was very regrettable that they could not be administered to the patient. The administration of $\mathrm{C} 1$-INH concentrate restores the inhibitory action of complement system by correcting the C1-INH deficiency or dysfunction. Also, C1INH concentrate is generally well-tolerated in the treatment of CLS after kidney transplantation caused by the preventive treatment of antibody-mediated rejection [11]. Daily intravenous infusions of C1-INH concentrate also showed effectiveness in patients with HAE accompanied by ascites, hypovolemic shock, and renal and respiratory failure who did not tolerate conventional ICU treatment [5]. Moreover, C1-INH concentrate is considered a multifunctional regulator of the cascade systems, which were recently shown to improve graft function by inhibiting complement activation and reducing the postoperative inflammation [10]. Our patient would have been better if he was treated with C1-INH concentrate. Fortunately, icatibant has recently become available in Korea and is thought to help severe HAE attacks like that of our patient because it can mediate vasodilatation and increase capillary permeability by preventing the receptor binding of bradykinin [7]. The use of FFP should only be considered when the firstline drugs are not available in an HAE attack. FFP is known as an effective substitute for C1-INH concentrate during
HAE because it contains C1-INH. However, FFP may worsen the symptoms paradoxically because it contains not only C1-INH but also other substrates such as kininogens [3]. Therefore, the anesthesiologist should monitor the patient's condition during an HAE attack treated with FFP. There is a possibility that the poor outcome of our patient despite the increase in $\mathrm{C} 1$-INH levels after the massive transfusion of FFP was due to the increase in harmful substrates administered in the FFP. Unfortunately, our patient had no option for treatment except FFP.

In conclusion, HAE is a disease with a decrease or dysfunction of C1-INH, which may lead to life-threatening complications such as CLS and DIC. Careful prophylactic therapy with androgen, FFP, and $\mathrm{Cl}$-INH concentrates is essential before surgery. However, in stressful conditions such as organ transplantation, HAE attacks may occur despite prophylactic treatment and produce fatal complications such as hypovolemic shock due to CLS and DIC. C1INH concentrate may be an important option for severe HAE attacks. We experienced a case of suspected CLS and DIC after kidney transplantation in a patient with HAE. Unfortunately, we could not use C1-INH concentrate for the patient at the appropriate time. In Korea, C1-INH concentrate is not available. However, the use of recently proved icatibant might help treat a severe HAE attack. If those first-line drugs are not available, FFP should be used with careful monitoring. The anesthesiologist should understand the critical complications of HAE and prepare the appropriate treatment options.

\section{CONFLICTS OF INTEREST}

No potential conflict of interest relevant to this article was reported.

\section{AUTHOR CONTRIBUTIONS}

Conceptualization: Ki Tae Jung. Data acquisition: Jeong Wook Park, Jinyoung Seo. Supervision: Ki Tae Jung. Writing-original draft: Jeong Wook Park, Jinyoung Seo, Ki Tae Jung. Writing-review \& editing: Sang Hun Kim, Ki Tae Jung.

\section{ORCID}

Jeong Wook Park, https://orcid.org/0000-0002-3990-4715

Jinyoung Seo, https://orcid.org/0000-0002-5256-6132 
Sang Hun Kim, https://orcid.org/0000-0003-3869-9470

Ki Tae Jung, https://orcid.org/0000-0002-2486-9961

\section{REFERENCES}

1. Zuraw BL. Clinical practice. Hereditary angioedema. N Engl J Med 2008; 359: 1027-36.

2. Vilaça MJL, Coelho FM, Faísco A, Carmona C. [Anesthetic considerations for a patient with hereditary angioedema - a clinical case]. Rev Bras Anestesiol 2017 67: 541-3. Portuguese.

3. Williams AH, Craig TJ. Perioperative management for patients with hereditary angioedema. Allergy Rhinol (Providence) 2015; 6: 50-5.

4. Cohen N, Sharon A, Golik A, Zaidenstein R, Modai D. Hereditary angioneurotic edema with severe hypovolemic shock. J Clin Gastroenterol 1993; 16: 237-9.

5. Pham H, Santucci S, Yang WH. Successful use of daily intravenous infusion of $\mathrm{Cl}$ esterase inhibitor concentrate in the treatment of a hereditary angioedema patient with ascites, hypovolemic shock, sepsis, renal and respiratory failure. Allergy Asthma Clin Immunol 2014; 10: 62.

6. Oguma K, Suzuki T, Mano S, Takeuchi S, Takeda J, Maruyama Y, et al. Hereditary angioedema with deep vein thrombosis and pulmonary thromboembolism during pregnancy. Taiwan J Obstet Gynecol 2019; 58: 895-6.

7. Maurer M, Magerl M, Ansotegui I, Aygören-Pürsün E, Betschel S, Bork K, et al. The international WAO/EAACI guideline for the management of hereditary angioedema-the 2017 revision and update. Allergy 2018; 73: 1575-96.

8. Levi M, Cohn DM, Zeerleder S. Hereditary angioedema: linking complement regulation to the coagulation system. Res Pract Thromb Haemost 2018; 3: 38-43.

9. Nielsen EW, Johansen HT, Gaudesen O, Osterud B, Olsen JO, Høgåsen K, et al. C3 is activated in hereditary angioedema, and $\mathrm{C} 1 / \mathrm{C} 1$-inhibitor complexes rise during physical stress in untreated patients. Scand J Immunol 1995; 42: 679-85.

10. Kirschfink M. C1-inhibitor and transplantation. Immunobiology 2002; 205: 534-41.

11. Ramirez-Sandoval JC, Varela-Jimenez R, Morales-Buenrostro LE. Capillary leak syndrome as a complication of antibody-mediated rejection treatment: a case report. CEN Case Rep 2018; 7: 110-3.

12. Kodama J, Uchida K, Kushiro H, Murakami N, Yutani C. Hereditary angioneurotic edema and thromboembolic diseases: I: how symptoms of acute attacks change with aging. Intern Med 1998; 37: 440-3. 